\title{
Treatment Outcomes and Prognostic Factors of Acute Variceal Bleeding in Patients with Hepatocellular Carcinoma
}

\author{
Yu Rim Lee, Soo Young Park, and Won Young Tak \\ Department of Internal Medicine, School of Medicine, Kyungpook National University, Kyungpook National University Hospital, Daegu, Korea
}

Background/Aims: The treatment outcomes and prognostic markers of acute variceal bleeding (AVB) in hepatocellular carcinoma (HCC) patients remain unclear. Therefore, we evaluated the clinical outcomes and prognostic factors of AVB in HCC patients. Methods: Cirrhotic patients with endoscopically confirmed AVB between 2007 and 2013 were enrolled in this prospective study. Prognostic factors were identified by multivariate Cox proportional hazards regression analysis. Results: Among the 329 enrolled patients, 125 patients (38.0\%) were diagnosed with HCC. The 6-week mortality rates of all enrolled AVB patients and the HCC subgroup were $14.9 \%$ and $26.4 \%$. The 5-day treatment failure, 6-week mortality, cirrhosis-related complications, and duration of hospitalization were greater in HCC patients than in non-HCC patients (all $\mathrm{p}<0.05$ ). In the HCC subgroup, the Model for End-Stage Liver Disease (MELD) score (hazard ratio [HR], 1.145; $p=0.001$ ) and Barcelona Clinic Liver Cancer (BCLC) stage (C-D vs 0-B) (HR, 3.096; $p=0.019)$ were independent predictors of 6-week mortality. Our study revealed that $85 \%$ of HCC patients with both a MELD score $\geq 15.5$ and BCLC stage C-D died within 6 weeks, and the 6-week mortality risk was 21-fold higher in this group than in the group with a lower MELD score and earlier HCC stage $(p<0.001)$. Conclusions: The 5-day treatment failure and 6-week mortality rates were significantly higher among AVB patients with HCC than those without HCC. The MELD score and the presence and stage of HCC are strong predictors of 6-week mortality in patients with AVB. (Gut Liver 2020;14:500-508)

Key Words: Hemorrhage; Esophageal and gastric varices; End stage liver disease; Carcinoma, hepatocellular; Mortality

\section{INTRODUCTION}

Acute variceal bleeding (AVB) is one of the most serious complications of liver cirrhosis (LC) and is associated with significant morbidity and mortality. ${ }^{1,2}$ The management of AVB has improved in the past decades, which includes the use of vasoactive drugs, prophylactic antibiotics, hemostatic procedures with endoscopy, and more recently, the salvage transjugular intrahepatic portosystemic shunt. ${ }^{3-5}$ Despite these advances in the management of AVB, it is still a life-threatening event and the 6-week mortality remains approximately $20 \%{ }^{4,6-9}$ Therefore, it is important to identify patients with a high mortality risk and tailor the treatment strategy according to the risk.

Various prognostic factors that can affect the rebleeding and mortality of AVB have been reported. Well-known prognostic factors include not only a hepatic venous pressure gradient of $\geq 20 \mathrm{mmHg}$ associated with portal hypertension and hematemesis at presentation, active bleeding at the time of endoscopy, and hypovolemic shock related to the severity of bleeding, but also hepatic encephalopathy, serum albumin level, bilirubin level, the Model for End-Stage Liver Disease (MELD) score, and the Child-Turcotte-Pugh score associated with the severity of cirrhosis. ${ }^{69-15}$ However, the impact of the presence and stage of hepatocellular carcinoma (HCC), which is frequently accompanied by advanced LC, on the outcome of variceal bleeding is not well known, and there are only few studies on the treatment outcomes and prognostic factors of AVB patients with HCC. ${ }^{12,16}$ The patients in these studies were not treated with the current standard protocol including the use of prophylactic antibiotics, vasoactive drugs, and endoscopic procedures. Moreover, these data have been obtained from retrospective studies with a relatively small number of patients. ${ }^{12,16}$ Therefore, further studies on the clinical outcome and factors associated with the prognosis

Correspondence to: Soo Young Park ${ }^{\mathrm{a}}$ and Won Young Tak ${ }^{\mathrm{b}}$

Department of Internal Medicine, School of Medicine, Kyungpook National University, Kyungpook National University Hospital, 130 Dongdeok-ro, Jung-gu, Daegu 41944, Korea

Tel: +82-53-200-5519, Fax: +82-53-426-8773, E-mail: psyoung0419@gmail.com

${ }^{\mathrm{b}}$ Tel: +82-53-200-5516, Fax: +82-53-426-8773, E-mail: wytak@knu.ac.kr

Received on May 7, 2019. Revised on August 8, 2019. Accepted on August 27, 2019. Published online December 11, 2019. pISSN 1976-2283 eISSN 2005-1212 https://doi.org/10.5009/gnl19155

@ This is an Open Access article distributed under the terms of the Creative Commons Attribution Non-Commercial License (http://creativecommons.org/licenses/by-nc/4.0) which permits unrestricted non-commercial use, distribution, and reproduction in any medium, provided the original work is properly cited. 
of AVB patients with HCC are required.

The MELD score has been used to estimate the prognosis of AVB. ${ }^{12,17,18}$ In comparison with the Child-Turcotte-Pugh score, which includes ascites and hepatic encephalopathy, the MELD score is more objective and accurate. A recent study has also revealed that a MELD-based model could precisely predict the 6-week mortality of AVB patients. ${ }^{19}$ However, the MELD score has been rarely validated in predicting the rebleeding and mortality of AVB patients with HCC.

We investigated the impact of HCC on the treatment outcome and prognostic factors of rebleeding and mortality of AVB patients, focusing on HCC patients.

\section{MATERIALS AND METHODS}

\section{Patients}

Patients with LC who visited Kyungpook National University Hospital, Daegu, South Korea between 2007 and 2013 due to AVB were consecutively enrolled in this prospective cohort study. Only patients with endoscopy-proven AVB were included in the study. AVB was defined as the spurting or oozing of blood from varices during endoscopy or when blood was observed in the esophagus or stomach with stigmata of recent bleeding on the varix..$^{9,20,21}$ Exclusion criteria were age under 20 years, severe or uncontrolled systemic illness, malignancy other than HCC, bleeding episodes within the previous 6 weeks, previous placement of a transjugular intrahepatic portosystemic shunt, and pregnancy (Fig. 1).

Diagnosis of HCC was based on historical data, pathologically or clinically using typical imaging studies such as computed tomography and/or magnetic resonance imaging according to the guideline of the European Association for the Study of the Liver. $^{22}$ The staging of HCC was made within 1 month of admission by contrast-enhanced abdominal computed tomography and/or magnetic resonance imaging using the Barcelona Clinic Liver Cancer (BCLC) classification. The hepatic reserve status was assessed based on the MELD score calculated from data taken within 12 hours from AVB.

The study was performed in accordance with the Helsinki Declaration of 1975, as revised in 2008 and approved by the Institutional Review Board of Kyungpook National University Hospital. Informed consent was obtained from all patients included in the study (IRB number: KNUH 2011-01-012).

\section{Management of variceal bleeding}

Management of variceal bleeding was performed according to Baveno IV-V recommendations. ${ }^{2,23}$ The patients were observed in the intensive care unit for at least 24 hours until the vital signs were stabilized. Upon admission, the patients were immediately resuscitated with fluid administration accompanied by blood sampling. Packed red blood cells were given to the patients to target hemoglobin level between 7 and $8 \mathrm{~g} / \mathrm{dL}$. A vasoactive drug (terlipressin or somatostatin) was administered at admission to all patients and continued for 3 days, and prophylactic antibiotics (ciprofloxacin or third-generation cephalosporin) were administered for 5 to 7 days. Endoscopic hemostasis, including endoscopic variceal band ligation or endoscopic variceal obturation with N-butyl-2-cyanoacrylate, was performed by highly experienced endoscopists within 24 hours of hospital admission. ${ }^{16}$ Varices were classified according to the Japanese Research Society for Portal Hypertension and the Esophagogastric Varices Grading System. ${ }^{24}$

\section{Clinical assessment and follow-up}

Historical and clinical information was obtained from the enrolled patients or their family members. Hemodynamic parameters were recorded from the time of visit, followed by every 4 hours for 48 hours and then every 8 hours. Laboratory tests

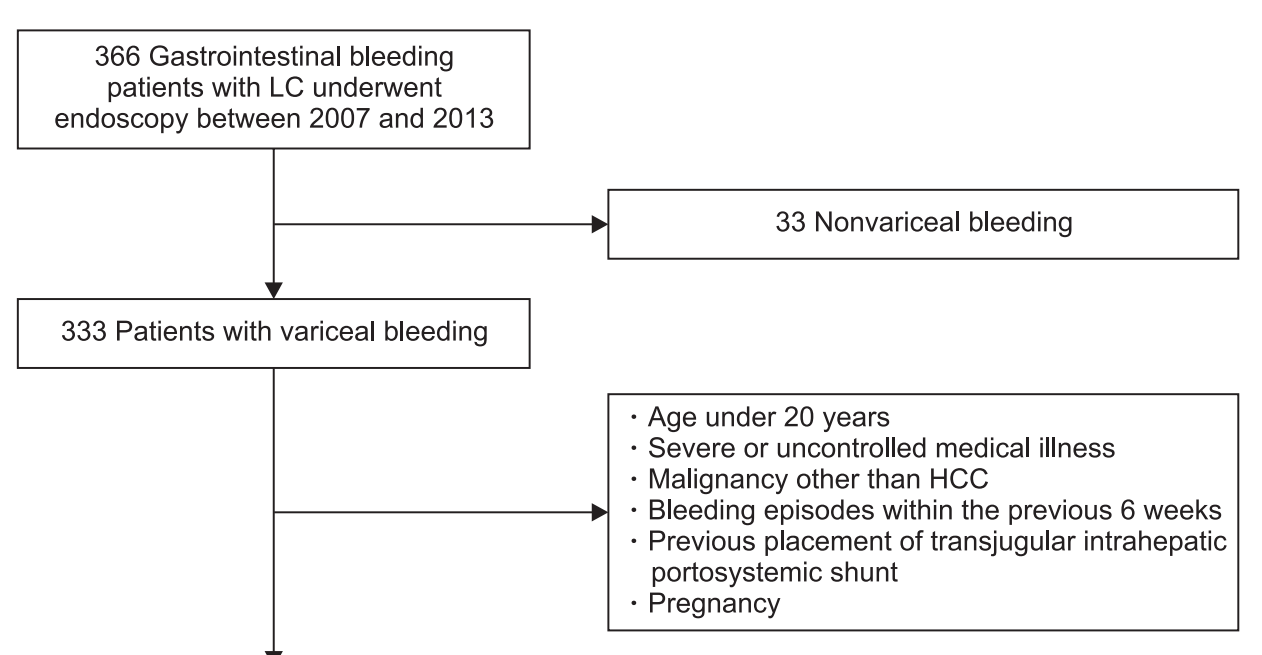

In total, 329 patients were analyzed
Fig. 1. Flowchart of patient enrollment. A total of 366 consecutive cirrhotic patients with upper gastrointestinal bleeding were suspected of having acute variceal bleeding and underwent endoscopy; of these, 329 patients met the inclusion criteria and were analyzed. LC, liver cirrhosis; HCC, hepatocellular carcinoma. 
were conducted for all patients on admission. Endoscopic findings were also documented, including bleeding source, varix size, active bleeding at the time of endoscopy, and outcome of endoscopic hemostasis. The door-to-endoscopy time was defined as the time interval from a presentation in the emergency room to when endoscopy was performed. The 5-day treatment failure (uncontrolled bleeding, rebleeding, or death) was defined based on Baveno IV-V criteria. ${ }^{2,23}$ Rebleeding was defined as the occurrence of bleeding symptoms including hematemesis, melena, or hematochezia, a decrease in hemoglobin, the requirement for transfusion of more than two units of packed red blood cells, or hemodynamic instability after 24 hours from achieving hemostasis with stable vital signs and hemoglobin. When treatment failure or rebleeding occurred, endoscopy was performed immediately to identify the bleeding focus and rescue hemostatic procedures including repeated endoscopic therapy and the placement of a transjugular intrahepatic portosystemic shunt were followed. Once the bleeding was controlled, the patients were treated with either nonselective beta-blockers or band ligation of varices as prophylaxis for the prevention of rebleeding. The clinical course, complications, and duration of hospital stay were recorded. All patients were followed regularly at our outpatient clinic until death or follow-up loss.

\section{Statistical analysis}

Data are presented as the median (interquartile range [IQR]) or number (\%) values as appropriate. Categorical variables were compared using the chi-square test (or Fisher exact test), while the Student t-test or Mann-Whitney test was used to compare continuous variables. The cumulative rebleeding and mortality rates were estimated using the Kaplan-Meier method. The factors associated with rebleeding and mortality were identified with the multivariate Cox proportional hazards regression test using variables that were significant in the univariate analysis. Considering the presence of statistical collinearity, individual variables required for calculating the MELD score were excluded from the multivariate analysis. This approach was also used in subgroup analyses. The optimal cutoff value was calculated as the value that maximized the sum of the sensitivity and specificity using time-dependent receiver operating characteristic curves and the area under the receiver operating characteristic curves (AUROCs). The hazards ratio (HR) and 95\% confidence interval (CI) were also determined. A probability value of $\mathrm{p}<0.05$ was considered to be statistically significant. Statistical analyses were performed using SPSS version 25.0 (PASW Inc., Chicago, IL, USA).

\section{RESULTS}

\section{Patients}

During the study period, a total of 366 consecutive cirrhotic patients with upper gastrointestinal bleeding who were suspected to have AVB underwent endoscopy. Thirty-three of these patients were excluded from the study because of nonvariceal bleeding. Of the 333 remaining patients with AVB, another four patients were excluded based on our exclusion criteria. Finally, 329 patients (125 HCC [38.0\%] and 204 LC without HCC [62.0\%]) were included in the statistical analyses (Fig. 1).

The baseline characteristics of the patients are shown in Table 1. The median age of the entire study population was 57 years (IQR, 51 to 67 years). The most common etiology of cirrhosis was hepatitis B ( $n=134,40.7 \%)$, followed by alcohol $(n=133$, 40.4\%). Hematemesis was present in 227 patients (69.0\%). The number of patients experiencing the first variceal bleeding episode was 197 (59.9\%), whereas 132 patients (40.1\%) had previous history of AVB. A total of 258 patients (78.4\%) had esophageal variceal hemorrhage, with 45 patients (13.7\%) having gastric variceal hemorrhage. Active bleeding was observed at the time of endoscopy in 218 patients (66.3\%). All patients (100\%) received vasoactive drug (terlipressin of $48.3 \%$ and somatostatin of 51.7\%), and prophylactic antibiotic therapy was administered to 111 patients (33.7\%). The median MELD score was 12 (IQR, 10 to 16). The number of patients who were receiving nonselective beta-blockers for the primary or secondary prophylaxis for variceal bleeding were 86,161 , respectively.

Among the 125 HCC patients, 40 patients had history of HCC without viable tumor. Stages of HCC patients were as follows: six with BCLC 0, 11 with BCLC A, eight with BCLC B, 31 with BCLC C, and 29 with BCLC D. The median duration from the diagnosis of HCC to AVB event was 6.0 months (IQR, 1.0 to 29.9 months). The baseline characteristics of HCC patients are shown in Table 1. Patients with HCC were significantly older (60.5 vs $56, p=0.01)$ and showed higher prevalence of hepatitis B $(60.8 \%$ vs $28.4 \%, \mathrm{p}<0.001)$ and proportion of first bleeding $(70.4 \%$ vs $53.4 \%, p=0.002)$. Patients with HCC also showed higher platelet count $(\mathrm{p}=0.042)$, higher aspartate aminotransferase level ( $\mathrm{p}=0.003)$, higher alanine aminotransferase (ALT) level ( $\mathrm{p}=0.033)$, significantly lower serum albumin level $(\mathrm{p}=0.021)$, and lower proportion with Child-Pugh class A $(\mathrm{p}=0.011)$ compared to patients without HCC. The median MELD score was not significantly different between patients with HCC and non-HCC group $(\mathrm{p}=0.178)$.

\section{Treatment outcomes of AVB}

Initial hemostasis was successfully achieved in 311 patients (94.5\%), which included 115 patients (92.0\%) with HCC and 196 patients $(96.1 \%)$ without HCC $(\mathrm{p}=0.114)$. Rebleeding occurred in 191 patients at a median of 2.8 months (IQR, 0.7 to 10.4 months) during follow-up period (15.6 months: IQR, 1.9 to 48.2 months). The 5-day treatment failure occurred in 33 patients, and rebleeding occurred in 66 patients within 6 weeks. The 5-day treatment failure and 6-week rebleeding were significantly higher among patients with HCC than among patients without HCC (17.6\% vs 5.4\%, p<0.001; 29.6\% vs $14.2 \%, p=0.001$ 
Table 1. Baseline Characteristics of the Study Population $(n=329)$

\begin{tabular}{|c|c|c|c|c|}
\hline Characteristic & All & HCC $(n=125,38.0 \%)$ & LC without HCC ( $n=204,62.0 \%)$ & p-value \\
\hline \multicolumn{5}{|l|}{ Demographic variable } \\
\hline Age, yr & $57(51-67)$ & $61(53-68)$ & $56(49-65)$ & 0.01 \\
\hline Male sex & $264(80.2)$ & $100(80.0)$ & $164(80.4)$ & 0.931 \\
\hline Etiology & & & & $<0.001$ \\
\hline HBV & $134(40.7)$ & $76(60.8)$ & $58(28.4)$ & \\
\hline $\mathrm{HCV}$ & $34(10.3)$ & $18(14.4)$ & $16(7.8)$ & \\
\hline Alcohol & $133(40.4)$ & $26(20.8)$ & $107(52.5)$ & \\
\hline Others & $28(8.5)$ & $5(4.0)$ & $23(11.3)$ & \\
\hline Hematemesis at the initial presentation & 227 (69.0) & $88(70.4)$ & $139(68.1)$ & 0.667 \\
\hline First bleeding episode & $197(59.9)$ & $88(70.4)$ & $109(53.4)$ & 0.002 \\
\hline Initial systolic blood pressure $<100 \mathrm{~mm} \mathrm{Hg}$ & $236(71.7)$ & $86(68.8)$ & $150(73.5)$ & 0.355 \\
\hline Heart rate $<100 /$ min & $202(61.4)$ & $84(67.2)$ & $118(57.8)$ & 0.091 \\
\hline \multicolumn{5}{|l|}{ Endoscopic variables } \\
\hline Door to endoscopy time, $\mathrm{hr}$ & $4(2-10)$ & $3(2-9)$ & $4(2-12)$ & 0.191 \\
\hline Bleeding cause & & & & 0.71 \\
\hline E & $258(78.4)$ & 97 (77.6) & 161 (78.9) & \\
\hline G & $45(13.7)$ & $15(12.0)$ & $30(14.7)$ & \\
\hline $\mathrm{E}+\mathrm{G}$ & $26(7.9)$ & $13(10.4)$ & $13(6.4)$ & \\
\hline Varix size & & & & 0.694 \\
\hline$\leq \mathrm{F} 1$ & $91(28.1)$ & $33(26.8)$ & $58(28.9)$ & \\
\hline$\geq \mathrm{F} 2$ & $233(71.9)$ & 90 (73.2) & $143(71.1)$ & \\
\hline Active bleeding on initial endoscopy & $218(66.3)$ & $79(63.2)$ & $139(68.1)$ & 0.358 \\
\hline Successful endoscopic hemostasis & $311(94.5)$ & $115(92.0)$ & $196(96.1)$ & 0.114 \\
\hline Vasoactive agent & & & & 0.051 \\
\hline Terlipressin & $159(48.3)$ & $69(55.2)$ & $90(44.1)$ & \\
\hline Somatostatin & $170(51.7)$ & $56(44.8)$ & $114(55.9)$ & \\
\hline PPI use & $159(48.3)$ & $66(52.8)$ & $93(45.6)$ & 0.204 \\
\hline Antibiotic prophylaxis & $111(33.7)$ & $44(35.2)$ & $67(32.8)$ & 0.661 \\
\hline \multicolumn{5}{|l|}{ Laboratory variables } \\
\hline Hemoglobin, g/dL & $8.9(7.5-10.5)$ & $9.1(7.6-10.525)$ & $8.8(7.3-10.4)$ & 0.464 \\
\hline WBC count, $\times 10^{3} / \mu \mathrm{L}$ & $7,050(4,960-10,450)$ & $6,960(5,015-10,547.5)$ & $7,100(4,900-10,450)$ & 0.947 \\
\hline Platelet count, $\times 10^{9} / \mathrm{L}$ & $98(65-135.5)$ & $102(63-155.75)$ & $97(65-128)$ & 0.042 \\
\hline Prothrombin time, sec & $15.2(13.6-17.2)$ & $15.05(13.575-16.7)$ & $15.4(13.8-17.25)$ & 0.428 \\
\hline Prothrombin time, INR & $1.39(1.24-1.54)$ & $1.39(1.24-1.53)$ & $1.39(1.25-1.55)$ & 0.795 \\
\hline Aspartate aminotransferase, IU/L & $49(32-104)$ & $56(37.5-137.8)$ & $43(29-89)$ & 0.003 \\
\hline Alanine aminotransferase, IU/L & $26(16-44.5)$ & $28.5(17.8-49.3)$ & $24(15-42)$ & 0.033 \\
\hline Total bilirubin, mg/dL & $1.59(0.92-2.78)$ & $1.64(0.99-2.75)$ & $1.57(0.86-2.78)$ & 0.28 \\
\hline Serum albumin, g/dL & $2.8(2.4-3.2)$ & $2.7(2.3-3.1)$ & $2.8(2.4-3.3)$ & 0.021 \\
\hline Creatinine, mg/dL & $0.85(0.70-1.10)$ & $0.87(0.71-1.23)$ & $0.83(0.68-1.05)$ & 0.131 \\
\hline Child-Pugh class & & & & 0.011 \\
\hline A & $69(21.0)$ & $17(13.5)$ & $52(25.6)$ & \\
\hline B & $190(57.8)$ & $77(61.1)$ & $113(55.7)$ & \\
\hline $\mathrm{C}$ & $70(21.3)$ & $32(25.4)$ & $38(18.7)$ & \\
\hline MELD score & $12(10-16)$ & $13(10-16)$ & $12(10-16)$ & 0.178 \\
\hline Presence of ascites & $207(62.9)$ & 85 (78.6) & $122(59.9)$ & 0.135 \\
\hline Presence of infection & $21(6.4)$ & $9(7.2)$ & $12(5.9)$ & 0.635 \\
\hline
\end{tabular}

Data are presented as median (interquartile range) or number (\%).

HCC, hepatocellular carcinoma; LC, liver cirrhosis; HBV, hepatitis B virus; HCV, hepatitis C virus; E, esophageal varix; G, gastric varix; PPI, proton pump inhibitor; WBC, white blood cell; INR, international normalized ratio; MELD, Model for End-Stage Liver Disease. 
Table 2. Treatment Outcomes of Variceal Bleeding $(n=329)$

\begin{tabular}{|c|c|c|c|c|}
\hline Variable & All & $\begin{array}{c}\text { HCC } \\
(n=125,38.0 \%)\end{array}$ & $\begin{array}{l}\text { LC without HCC } \\
(n=204,62.0 \%)\end{array}$ & p-value \\
\hline Initial hemostasis achievement & $311(94.5)$ & $115(92.0)$ & $196(96.1)$ & 0.114 \\
\hline Rebleeding & $191(58.1)$ & $75(60.0)$ & 116 (56.9) & 0.576 \\
\hline Treatment failure or rebleeding within 5 day & $33(10.0)$ & $22(17.6)$ & $11(5.4)$ & $<0.001$ \\
\hline Rebleeding within 6 wk & $66(20.1)$ & $37(29.6)$ & $29(14.2)$ & 0.001 \\
\hline Death & $186(56.5)$ & 97 (77.6) & $89(43.6)$ & $<0.001$ \\
\hline In hospital mortality & $40(12.2)$ & $25(20.0)$ & $15(7.4)$ & 0.001 \\
\hline Mortality within $6 \mathrm{wk}$ & $49(14.9)$ & $33(26.4)$ & $16(7.8)$ & $<0.001$ \\
\hline PRBC transfusion, unit & $2(1-4)$ & $2(1-4)$ & $2(1-4)$ & 0.363 \\
\hline FFP transfusion, unit & $0(0-0)$ & $0(0-0)$ & $0(0-0)$ & 0.574 \\
\hline \multicolumn{5}{|l|}{ Cirrhosis-related complications } \\
\hline Hepatic failure & $26(8.0)$ & $18(14.5)$ & 8 (3.9) & 0.001 \\
\hline Hepatorenal syndrome & $16(4.9)$ & $11(8.9)$ & $5(2.5)$ & 0.009 \\
\hline Hepatic encephalopathy & $43(13.1)$ & 22 (17.7) & $21(10.3)$ & 0.055 \\
\hline Hospital stay, day & $5(3-9)$ & $6(3-13)$ & $5(3-8)$ & 0.001 \\
\hline
\end{tabular}

Data are presented as number (\%) or median (interquartile range).

HCC, hepatocellular carcinoma; LC, liver cirrhosis; PRBC, packed red blood cell; FFP, fresh frozen plasma.

respectively) (Table 2).

A total of 186 patients (56.5\%) died at a median of 4.3 months (IQR, 1.2 to 19.1 months). The mortality rate of patients with AVB was 14.9\% $(n=49)$ at 6 weeks. A significantly higher mortality rate within 6 weeks was observed among patients with HCC compared with those without HCC (26.4\% vs $7.8 \%$, $\mathrm{p}<0.001)$. The most common cause of death was bleeding $(n=66,35.5 \%)$, followed by hepatic failure ( $n=32,17.2 \%)$, infection $(n=11)$, hepatorenal syndrome $(n=10)$, and worsening of HCC $(n=8)$. In HCC group, the most common cause of death was bleeding $(n=35,36.1 \%)$, followed by hepatic failure $(n=18$, $18.6 \%)$, worsening of HCC $(n=7)$, infection $(n=5)$, and hepatorenal syndrome $(n=5)$. In non-HCC group, the most common cause of death was bleeding $(\mathrm{n}=31,34.8 \%)$, followed by hepatic failure $(n=14,15.7 \%)$, infection $(n=6)$, hepatorenal syndrome $(n=5)$, and worsening of HCC $(n=1)$. Cirrhosis-related complications such as hepatic failure, hepatorenal syndrome, and hepatic encephalopathy developed in 26, 16, and 43 patients, respectively. Hepatic failure (14.5\% vs 3.9\%, $\mathrm{p}=0.001$ ) and hepatorenal syndrome ( $8.9 \%$ vs $2.5 \%, \mathrm{p}=0.009$ ) were higher and the hospital stay was longer among patients with HCC than among patients without HCC. The median hospital stay was 6 days (IQR, 3 to 13 days) in the HCC patients and 5 days (IQR, 3 to 8 days) in patients without HCC ( $p=0.001)$ (Table 2). A total of four patients underwent HCC treatment during hospitalization. After excluding these four patients, the hospital stay was still longer in AVB patients with HCC than those without HCC ( 6 days [IQR, 3 to 12 days] vs 5 days [IQR, 3 to 8 days], $\mathrm{p}=0.002$ ). The cumulative rebleeding rates were $21.6 \%, 32.8 \%, 54.4 \%$, and $74.2 \%$ at 6 weeks, 3 months, 1 year, and 3 years, respectively; the corre- sponding cumulative mortality rates were 15.5\%, 25.9\%, 37.8\%, and $54.1 \%$, respectively. The rebleeding and mortality rate were significantly higher in the HCC group than in the non-HCC group (log-rank test $\mathrm{p}=0.001, \mathrm{p}<0.001$, respectively) (Fig. 2).

\section{Independent predictors of 5-day treatment failure and 6-week mortality in patients with AVB}

The results of univariate analysis and subsequent multivariate analysis are presented in Supplementary Tables 1 and 2 . The 5-day treatment failure was significantly associated with age (HR, $1.047 ; 95 \%$ CI, 1.013 to $1.082 ; \mathrm{p}=0.006$ ), active bleeding on initial endoscopy (HR, 2.343; 95\% CI, 1.074 to 5.115 ; $\mathrm{p}=0.032$ ), endoscopic hemostasis failure ( $\mathrm{HR}, 3.555 ; 95 \% \mathrm{CI}$, 1.518 to 8.326; $\mathrm{p}=0.003$ ), presence of ascites ( $\mathrm{HR}, 3.615$; 95\% $\mathrm{CI}, 1.071$ to $12.204 ; \mathrm{p}=0.038)$, and hepatorenal syndrome (HR, 3.228; 95\% CI, 1.169 to 8.914; p=0.024). Age (HR, 1.037; 95\% CI, 1.008 to $1.066 ; \mathrm{p}=0.011$ ), low initial systolic blood pressure (HR, 2.592; 95\% CI, 1.264 to 5.315; $\mathrm{p}=0.009$ ), a large varix (HR, 3.833; 95\% CI, 1.634 to 8.995; $\mathrm{p}=0.002$ ), endoscopic hemostasis failure (HR, 5.342; 95\% CI, 2.422 to 11.785 ; $\mathrm{p}<0.001$ ), ALT level (HR, 1.002; 95\% CI, 1.001 to $1.004 ; \mathrm{p}=0.01$ ), MELD score (HR, $1.111 ; 95 \% \mathrm{CI}, 1.042$ to $1.184 ; \mathrm{p}=0.001)$, presence of HCC (HR, 2.915; 95\% CI, 1.506 to 5.642; $\mathrm{p}=0.001$ ), and hepatic failure (HR, 3.038; 95\% CI, 1.540 to $5.991 ; \mathrm{p}=0.001$ ) were all significantly associated with the 6-week mortality after AVB in multivariate analysis. The increase in MELD score between the time of admission and 1-week or 1-month follow-up were also significantly associated with 6-week mortality (HR, 1.194; 95\% CI, 1.136 to $1.255 ; \mathrm{p}<0.001$; HR, $1.271 ; 95 \%$ CI, 1.178 to 1.372 ; $\mathrm{p}<0.001$, respectively). 

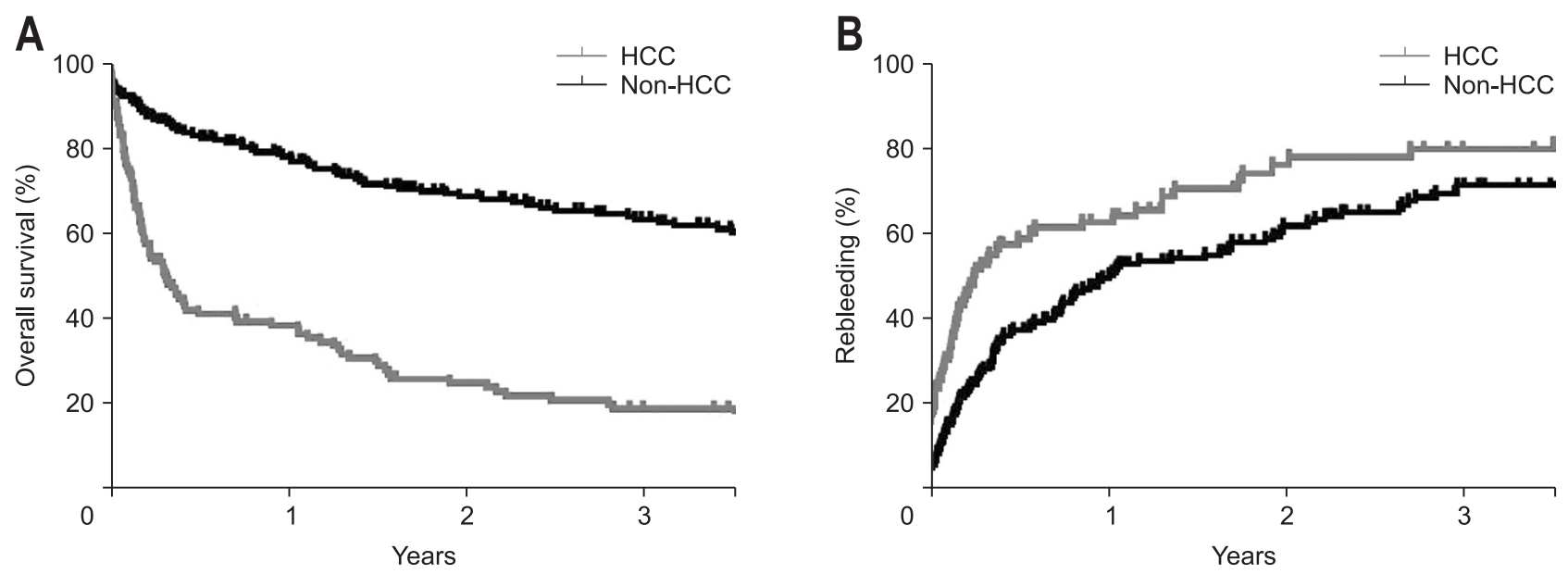

Fig. 2. Kaplan-Meier plots. (A) Overall survival according to the hepatocellular carcinoma (HCC) group ( $\mathrm{n}=125)$ versus non-HCC group ( $\mathrm{n}=204)$. The survival rate was significantly lower for patients with HCC than for non-HCC patients (log-rank test $\mathrm{p}<0.001)$. (B) Cumulative rebleeding according to the HCC group $(n=125)$ versus non-HCC group $(n=204)$. The rebleeding rate was significantly higher in patients with HCC than in those without HCC (log-rank test $\mathrm{p}=0.001)$.

Table 3. Independent Risk Factors for 5-Day Treatment Failure in the HCC Subgroup with Acute Variceal Hemorrhage $(\mathrm{n}=125)$

\begin{tabular}{|c|c|c|c|c|c|}
\hline \multirow{2}{*}{ Variable } & \multirow{2}{*}{$\begin{array}{c}\text { Univariate } \\
\text { p-value }\end{array}$} & \multicolumn{2}{|c|}{$\begin{array}{l}\text { Multivariate using HCC stage } \\
\text { (history of HCC, BCLC 0-B vs BCLC C-D) }\end{array}$} & \multicolumn{2}{|c|}{$\begin{array}{l}\text { Multivariate using HCC stage } \\
\text { (history of HCC, BCLC 0-C vs BCLC D) }\end{array}$} \\
\hline & & p-value & HR (95\% CI) & $\mathrm{p}$-value & HR $(95 \%$ CI) \\
\hline Endoscopic hemostasis failure & $<0.001$ & 0.049 & 2.907 (1.006-8.399) & 0.031 & $3.538(1.123-11.148)$ \\
\hline Alpha-fetoprotein, ng/mL & 0.024 & & & & \\
\hline PRBC transfusion & 0.05 & & & & \\
\hline PPI use & 0.06 & & & & \\
\hline MELD score & 0.003 & & & & \\
\hline HCC stage, BCLC stage (C-D vs 0-B) & 0.001 & 0.016 & $13.096(1.625-105.541)$ & 0.006 & $5.116(1.595-16.408)$ \\
\hline Presence of PVT & 0.001 & & & & \\
\hline Presence of ascites & 0.034 & & & & \\
\hline Hepatic failure & $<0.001$ & & & & \\
\hline Hepatorenal syndrome & 0.003 & & & & \\
\hline Hepatic encephalopathy & 0.022 & & & & \\
\hline
\end{tabular}

HCC, hepatocellular carcinoma; BCLC, Barcelona Clinic Liver Cancer; HR, hazard ratio; CI, confidence interval; PRBC, packed red blood cell; PPI, proton pump inhibitor; MELD, Model for End-Stage Liver Disease; PVT, portal vein thrombosis.

\section{Independent predictors of 5-day treatment failure and 6-week mortality in the HCC subgroup with AVB}

As shown in Table 3, higher 5-day treatment failure rate was observed in cases with endoscopic hemostasis failure (HR, 2.907; 95\% CI, 1.006 to 8.399; $\mathrm{p}=0.049$ ) and advanced BCLC stage (CD vs 0-B) (HR, 13.096; 95\% CI, 1.625 to $105.541 ; \mathrm{p}=0.016)$.

Endoscopic hemostasis failure (HR, 4.838; 95\% CI, 1.741 to 11.033; $\mathrm{p}=0.002$ ), MELD score (HR, 1.145; 95\% CI, 1.055 to 1.243; $\mathrm{p}=0.001$ ), and advanced BCLC stage ( $\mathrm{C}-\mathrm{D}$ vs $0-\mathrm{B})$ (HR, 3.096; 95\% CI, 1.204 to $7.960 ; \mathrm{p}=0.019$ ) were independent predictors of 6-week mortality in multivariate analysis (Table 4). The recalibrated MELD-based score was calculated as described by Reverter et al.: ${ }^{19}-5.312+0.207 \times$ MELD. The accuracy of the
MELD score and recalibrated MELD-based score in predicting the 6-week mortality of HCC patients after AVB was evaluated; the AUROC was 0.771 for both the MELD score and recalibrated MELD-based score. The increase in MELD score between the time of admission and 1-week or 1-month after admission were also significantly associated with 6-week mortality (HR, 1.138; 95\% CI, 1.061 to $1.221 ; \mathrm{p}<0.001$; HR, $1.224 ; 95 \% \mathrm{CI}, 1.127$ to 1.329, $\mathrm{p}<0.001$, respectively). HCC patients with viable tumors showed higher 5-day treatment failure and 6-week mortality than patients with HCC history without residual tumors ( $p=0.033$, $\mathrm{p}=0.020$, respectively). The presence of portal vein thrombosis (PVT) significantly affected the rates of 5-day treatment failure and 6-week mortality in the HCC subgroup ( $p=0.001, p<0.001$, respectively); considering the presence of statistical collinearity, 
Table 4. Independent Risk Factors for 6-Week Mortality in the HCC Subgroup with Acute Variceal Hemorrhage (n=125)

\begin{tabular}{|c|c|c|c|c|c|}
\hline \multirow{2}{*}{ Variable } & \multirow{2}{*}{$\begin{array}{c}\text { Univariate } \\
\text { p-value }\end{array}$} & \multicolumn{2}{|c|}{$\begin{array}{l}\text { Multivariate using HCC stage } \\
\text { (history of HCC, BCLC 0-B vs BCLC C-D) }\end{array}$} & \multicolumn{2}{|c|}{$\begin{array}{l}\text { Multivariate using HCC stage } \\
\text { (history of HCC, BCLC 0-C vs BCLC D) }\end{array}$} \\
\hline & & $\mathrm{p}$-value & HR (95\% CI) & $\mathrm{p}$-value & HR $(95 \%$ CI) \\
\hline Heart rate $<100 / \mathrm{min}$ & 0.015 & & & & \\
\hline Active bleeding on initial endoscopy & 0.038 & & & & \\
\hline Endoscopic hemostasis failure & $<0.001$ & 0.002 & 4.838 (1.741-11.033) & 0.001 & $4.136(1.750-9.773)$ \\
\hline Alanine aminotransferase, IU/L & 0.048 & & & & \\
\hline PPI use & 0.023 & & & & \\
\hline MELD score & $<0.001$ & 0.001 & $1.145(1.055-1.243)$ & 0.005 & $1.125(1.036-1.221)$ \\
\hline HCC stage, BCLC stage (C-D vs 0-B) & $<0.001$ & 0.019 & $3.096(1.204-7.960)$ & $<0.001$ & $5.129(2.312-1.380)$ \\
\hline Presence of PVT & $<0.001$ & & & & \\
\hline Hepatic failure & $<0.001$ & & & & \\
\hline Hepatorenal syndrome & 0.001 & & & & \\
\hline Hepatic encephalopathy & 0.015 & & & & \\
\hline
\end{tabular}

HCC, hepatocellular carcinoma; BCLC, Barcelona Clinic Liver Cancer; HR, hazard ratio; CI, confidence interval; PPI, proton pump inhibitor; MELD, Model for End-Stage Liver Disease; PVT, portal vein thrombosis.

Table 5. Six-Week Mortality of HCC Patients According to the MELD Score and BCLC Stage $(n=125)$

\begin{tabular}{lcc}
\hline Variable & p-value & HR (95\% CI) \\
\hline $\begin{array}{l}\text { MELD }<15.5 \& \\
\text { (history of HCC, BCLC 0-B) }\end{array}$ & - & Reference \\
MELD $\geq 15.5$ \& & 0.072 & 3.950 (0.883-17.658) \\
$\quad$ (history of HCC, BCLC 0-B) & & \\
MELD $<15.5$ \& BCLC stage C-D & 0.035 & $3.542(1.091-11.505)$ \\
MELD $\geq 15.5$ \& BCLC stage C-D & $<0.001$ & 21.724 (7.213-65.433) \\
\hline
\end{tabular}

HCC, hepatocellular carcinoma; MELD, Model for End-Stage Liver Disease; BCLC, Barcelona Clinic Liver Cancer; HR, hazard ratio; CI, confidence interval.

this variable was excluded from the multivariate analysis. The 6-week mortality rate was not different according to the position of PVT (main vs branch) ( $\mathrm{p}=0.763$ ).

The patients were stratified into four groups according to a MELD score of $<15.5$ or $\geq 15.5$ and early (BCLC stage $0-B$ ) or advanced (BCLC stage C-D) HCC (Table 5). Compared with patients with a lower MELD score $(<15.5)$ and earlier BCLC stage (0-B), those with a higher MELD score $(\geq 15.5)$ or advanced BCLC stage (C-D) alone, and those with both a higher MELD score $(\geq 15.5)$ and advanced BCLC stage (C-D) had an increased risk of 6-week mortality, with the latter two being statistically significant (both $\mathrm{p}<0.05$ ). The 6 -week mortality risk was 21-fold higher among patients with a higher MELD score ( $\geq 15.5)$ and advanced BCLC stage (C-D) than among those with a lower MELD score $(<15.5)$ and earlier BCLC stage $(0-$ B) $(p<0.001)$. The survival curves of the four groups until 6 weeks are shown in Fig. 3. Around 85\% of patients with both MELD score $\geq 15.5$ and BCLC stage C-D died within 6 weeks.
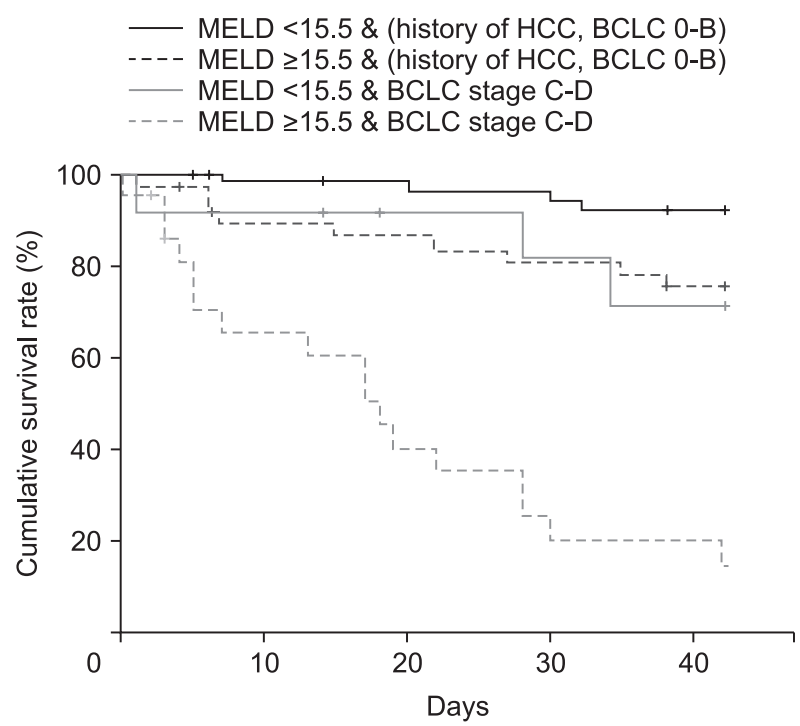

Fig. 3. Cumulative survival rates at 6 weeks based on the MELD score and BCLC stage. The risk of 6-week mortality was significantly higher among patients with a MELD score $\geq 15.5$ and BCLC stage C-D than among patients with a lower MELD score and earlier BCLC stage (logrank test $\mathrm{p}<0.001$ ).

MELD, Model for End-Stage Liver Disease; BCLC, Barcelona Clinic Liver Cancer.

\section{DISCUSSION}

AVB is associated with poor prognosis in patients with LC, thus it is important to identify the relevant prognostic factors. HCC, commonly accompanied by AVB, is known as a poor prognostic factor in AVB patients. ${ }^{6,12}$ However, there are limited studies on the treatment outcomes and prognostic factors of AVB in patients with different stage of HCC. Therefore, we investigated the treatment outcomes and prognostic factors of 
cirrhotic patients with AVB with a focus on HCC patients.

Among all enrolled AVB patients, 5-day treatment failure occurred in 33 patients (10.0\%), and 49 patients (14.9\%) died within 6 weeks, which is similar to previous findings. ${ }^{6,7,9}$ HCC patients showed higher rebleeding rate, mortality rate, cirrhosisrelated complications, and longer hospital stay than non-HCC patients. For AVB patients with HCC, endoscopic hemostasis failure, MELD score, and BCLC stage were independent predictors of 6-week mortality. The AUROC was 0.771 for both the MELD score and recalibrated MELD-based score in predicting 6-week mortality of AVB. We also found that the risk of 6-week mortality was 21-fold higher among patients with MELD score $\geq 15.5$ and advanced BCLC stage (C-D) than among those with a lower MELD score and earlier BCLC stage.

This study has several strengths and provides some important findings. First, it was a prospective cohort study enrolling a relatively large number of patients with AVB. Moreover, vasoactive drugs were used for all enrolled patients in accordance with recent guidelines. Second, we analyzed the impact of HCC with various stages and PVT on clinical outcomes in AVB patients, which have rarely been investigated. We found that the 5-day treatment failure and 6-week mortality were higher in the HCC group than in the non-HCC group. Moreover, the BCLC stage was an independent predictor of the 5-day treatment failure and 6-week mortality of AVB patients with HCC. Third, the MELD score, which is an accurate and objective variable, was used as a strong predictor of the 6-week mortality of all enrolled AVB patients and the HCC subgroup in this study. In previous studies, the MELD score has been demonstrated to estimate the prognosis of AVB and 3-month mortality of patients with decompensated liver disease. ${ }^{12,17}$ However, the MELD score as a predictor of mortality in variceal bleeding patients with HCC has been studied only in limited reports. In our study, both the MELD score and recalibrated MELD-based score accurately predicted the 6-week mortality of AVB patients with HCC (AUROC=0.771). The 6-week mortality rates were $11.1 \%$ and $31.0 \%$ for patients with MELD score $<15.5$ and $\geq 15.5$, respectively. Considering the high mortality of patients with MELD score $\geq 15.5$, the early identification and prophylactic treatment of large varices might be beneficial for these patients. Fourth, there has been no wellestablished model for prediction of mortality in AVB patients with HCC. In this study, 85\% of AVB patients with HCC having both a high MELD score $(\geq 15.5)$ and advanced cancer stage (BCLC C-D) died within 6 weeks, and these patients had a 21fold increased risk of 6-week mortality compared with patients with a lower MELD score $(<15.5)$ and earlier BCLC stage $(0-B)$. Therefore, these factors could be used in a prediction model of 6-week mortality for AVB patients with HCC.

The median MELD score were 12 (IQR, 10 to 16) at the time of admission, 12.0 (IQR, 9.7 to 15.0) at 1 week later, and 10.7 (IQR, 9.1 to 14.4 ) at 1 month later. The increase in MELD score between the time of admission and 1-week or 1-month follow- up were significantly associated with 6-week mortality in both all enrolled AVB patients and the HCC subgroup. Patients with high MELD score $(\geq 15.5)$ and/or MELD score worsening after 1 week or 1 month have a poor prognosis, so the possibility of liver transplantation should be considered in patients who are eligible to liver transplantation.

The presence of PVT significantly affected the rates of 5-day treatment failure and 6-week mortality in the HCC subgroup, which is similar to the findings of previous studies showing PVT as a prognostic factor of AVB. ${ }^{25}$ The 6-week mortality rate was not different according to the position of PVT (main vs branch). According to these results, the poor prognosis of AVB patients with HCC may be related with elevated portal pressure. However, only 55 patients had HCC concurrently with PVT in our study, so further studies are needed.

We found no clear association between the presence of active bleeding and the risk of 6-week mortality in all enrolled AVB patients and the HCC subgroup in multivariate analysis in contrast to previous studies. ${ }^{6}$ In addition, our study showed that the administration of a proton pump inhibitor had no effect on the 5-day treatment failure or 6-week mortality of AVB patients in multivariate analysis, which differs from previous findings. ${ }^{26}$ Since there have been reports of the risk of infection with longterm proton pump inhibitor use, ${ }^{27}$ further studies with a large number of patients and longer period are needed.

This study has several limitations. First, we did not measure the hepatic venous pressure gradient of enrolled AVB patients, so we could not investigate the prognostic ability of hepatic venous pressure gradient for mortality and rebleeding of AVB. Second, prophylactic antibiotics recommended in the current guideline were not used for all patients, and so follow-up studies are expected.

In conclusion, the 5-day treatment failure, 6-week mortality, and cirrhosis-related complications of AVB were significantly higher among patients with HCC compared with those without HCC. In addition, our study revealed that the risk of mortality was different according to the MELD score and the presence and stage of HCC in patients with AVB. These factors could allow the early identification of patients with increased mortality risk, and these patients may require more intensive care and frequent follow-up during the course of AVB.

\section{CONFLICTS OF INTEREST}

No potential conflict of interest relevant to this article was reported.

\section{AUTHOR CONTRIBUTIONS}

Study conception and design, acquisition of data, analysis and interpretation of data: Y.R.L., S.Y.P., W.Y.T. Drafting of the manuscript, statistical analysis: Y.R.L. Critical revision of the manu- 
script for important intellectual content, study supervision: S.Y.P., W.Y.T. All authors have approved the final draft submitted.

\section{ORCID}

Yu Rim Lee

Soo Young Park

Won Young Tak

\section{REFERENCES}

1. Graham DY, Smith JL. The course of patients after variceal hemorrhage. Gastroenterology 1981;80:800-809.

2. De Franchis R; Baveno V Faculty. Revising consensus in portal hypertension: report of the Baveno $\mathrm{V}$ consensus workshop on methodology of diagnosis and therapy in portal hypertension. $\mathrm{J}$ Hepatol 2010;53:762-768.

3. Chalasani N, Kahi C, Francois F, et al. Improved patient survival after acute variceal bleeding: a multicenter, cohort study. Am J Gastroenterol 2003;98:653-659.

4. Carbonell N, Pauwels A, Serfaty L, et al. Improved survival after variceal bleeding in patients with cirrhosis over the past two decades. Hepatology 2004;40:652-659.

5. García-Pagán JC, Caca K, Bureau C, et al. Early use of TIPS in patients with cirrhosis and variceal bleeding. N Engl J Med 2010; 362:2370-2379.

6. Jung JW. Role of endoscopic treatment or balloon-occluded retrograde transvenous obliteration in patients with Child-Pugh class C end-stage liver cirrhosis and esophageal/gastric varices. Clin Mol Hepatol 2019;25:181-182.

7. Garcia-Tsao G, Bosch J. Management of varices and variceal hemorrhage in cirrhosis. N Engl J Med 2010;362:823-832.

8. Jairath V, Rehal S, Logan R, et al. Acute variceal haemorrhage in the United Kingdom: patient characteristics, management and outcomes in a nationwide audit. Dig Liver Dis 2014;46:419-426.

9. Bambha K, Kim WR, Pedersen R, Bida JP, Kremers WK, Kamath PS. Predictors of early re-bleeding and mortality after acute variceal haemorrhage in patients with cirrhosis. Gut 2008;57:814820.

10. Abraldes JG, Villanueva C, Bañares R, et al. Hepatic venous pressure gradient and prognosis in patients with acute variceal bleeding treated with pharmacologic and endoscopic therapy. J Hepatol 2008;48:229-236.

11. Augustin S, Muntaner L, Altamirano JT, et al. Predicting early mortality after acute variceal hemorrhage based on classification and regression tree analysis. Clin Gastroenterol Hepatol 2009;7: 1347-1354.

12. Amitrano L, Guardascione MA, Bennato R, Manguso F, Balzano A. MELD score and hepatocellular carcinoma identify patients at different risk of short-term mortality among cirrhotics bleeding from esophageal varices. J Hepatol 2005;42:820-825.

13. Chen PH, Chen WC, Hou MC, et al. Delayed endoscopy increases re-bleeding and mortality in patients with hematemesis and active esophageal variceal bleeding: a cohort study. J Hepatol 2012;57:1207-1213.

14. Augustin S, Altamirano J, González A, et al. Effectiveness of combined pharmacologic and ligation therapy in high-risk patients with acute esophageal variceal bleeding. Am J Gastroenterol 2011; 106:1787-1795.

15. Lecleire S, Di Fiore F, Merle V, et al. Acute upper gastrointestinal bleeding in patients with liver cirrhosis and in noncirrhotic patients: epidemiology and predictive factors of mortality in a prospective multicenter population-based study. J Clin Gastroenterol 2005;39:321-327.

16. Han ML, Chen CC, Kuo SH, et al. Predictors of in-hospital mortality after acute variceal bleeding in patients with hepatocellular carcinoma and concurrent main portal vein thrombosis. J Gastroenterol Hepatol 2014;29:344-351.

17. Wiesner R, Edwards E, Freeman R, et al. Model for end-stage liver disease (MELD) and allocation of donor livers. Gastroenterology 2003;124:91-96.

18. Chalasani N, Kahi C, Francois F, et al. Model for End-Stage Liver Disease (MELD) for predicting mortality in patients with acute variceal bleeding. Hepatology 2002;35:1282-1284.

19. Reverter E, Tandon P, Augustin S, et al. A MELD-based model to determine risk of mortality among patients with acute variceal bleeding. Gastroenterology 2014;146:412-419.

20. Sarin SK, Kumar A, Angus PW, et al. Diagnosis and management of acute variceal bleeding: Asian Pacific Association for Study of the Liver recommendations. Hepatol Int 2011;5:607-624.

21. Hou MC, Lin HC, Kuo BI, Lee FY, Schmidt CM, Lee SD. Clinical implications of the white nipple sign and its role in the diagnosis of esophageal variceal hemorrhage. Am J Gastroenterol 1996;91: 2103-2109.

22. Forner A, Llovet JM, Bruix J. Hepatocellular carcinoma. Lancet 2012;379:1245-1255.

23. De Franchis R. Evolving consensus in portal hypertension: report of the Baveno IV consensus workshop on methodology of diagnosis and therapy in portal hypertension. J Hepatol 2005;43:167-176.

24. Idezuki Y. General rules for recording endoscopic findings of esophagogastric varices (1991). Japanese Society for Portal Hypertension. World J Surg 1995;19:420-423.

25. Amitrano L, Guardascione MA, Manguso F, et al. The effectiveness of current acute variceal bleed treatments in unselected cirrhotic patients: refining short-term prognosis and risk factors. Am J Gastroenterol 2012;107:1872-1878.

26. Lo GH, Perng DS, Chang CY, Tai CM, Wang HM, Lin HC. Controlled trial of ligation plus vasoconstrictor versus proton pump inhibitor in the control of acute esophageal variceal bleeding. J Gastroenterol Hepatol 2013;28:684-689.

27. Lo EA, Wilby KJ, Ensom MH. Use of proton pump inhibitors in the management of gastroesophageal varices: a systematic review. Ann Pharmacother 2015;49:207-219. 